\title{
Estimativa de Desempenho Acadêmico de Estudantes: Análise da Aplicação de Técnicas de Mineração de Da- dos em Cursos a Distância
}

Title: Estimating Academic Student Performance: Analyzing Application of Data Mining Techniques in Distance Learning

\author{
Ernani Gottardo \\ Instituto Federal de Educação, Ciência e Tecnologia do RS \\ - IFRS \\ CEP 99.700-000 - Erechim - RS - Brasil \\ ernani.gottardo@erechim.ifrs.edu.br \\ Robinson Vida Noronha \\ Universidade Tecnológica Federal do Paraná (UTFPR) \\ CEP 80.230-901 - Curitiba - PR - Brasil. \\ vida@utfpr.edu.br
}

\author{
Celso Antonio Alves Kaestner \\ Universidade Tecnológica Federal do Paraná (UTFPR) \\ CEP 80.230-901 - Curitiba - PR - Brasil. \\ celsokaestner@utfpr.edu.br
}

\begin{abstract}
Resumo Ambientes educacionais têm incorporado o uso de Softwares para apoio às atividades de ensino. Para o desenvolvimento de cursos a distância a utilização de um Ambiente Virtual de Aprendizagem (AVA) tornou-se fundamental. Um AVA normalmente armazena grandes volumes de dados, registrando detalhadamente as atividades desenvolvidas pelos estudantes. Esses dados podem ser utilizados para a descoberta de informações que auxiliem professores na gestão do processo de ensino-aprendizagem. Neste trabalho, através da utilização de técnicas de mineração de dados, investiga-se como obter inferências relativas ao desempenho de estudantes em cursos a distância baseando-se em dados obtidos de um Ambiente Virtual de Aprendizagem. Experimentos realizados demonstraram a viabilidade desta proposta, atingindo-se indices entre $73 \%$ e $80 \%$ de acerto nas estimativas de desempenho acadêmico dos estudantes.
\end{abstract}

Palavras-Chave: Mineração de Dados Educacionais, Educação a Distância, Ambientes Virtuais de Aprendizagem, Estimativa de Desempenho de Estudantes

\begin{abstract}
Educational environments have incorporated the use of software to support learning activities. A Learning Management System (LMS) is essential to dispatch distance learning courses to students. A LMS typically stores large volumes of data, recording in detail the activities performed by students. These data can be used to discover relevant information that can help teachers in the management of the teaching-learning process. In this study, by using data mining techniques, we investigate how to obtain inferences about the performance of students in distance learning courses based on data obtained from a Learning Management System. Some experiments conducted in this research indicate the viability of this proposal, achieving accuracy rates between $73 \%$ and $80 \%$ in estimates students' academic performance.
\end{abstract}

Keywords: Educacional Data Mining, Distance Learning Education, Virtual Learning Environments, Students' Performance Prediction 


\section{Introdução}

Ambientes Educacionais têm se caracterizado pelo crescente uso de softwares de apoio à atividade de ensino-aprendizagem. Destacam-se dentre esses softwares os Ambientes Virtuais de Aprendizagem (AVA) e os Sistemas Tutores Inteligentes ou ITS (Intelligent Tutoring System). Esses sistemas, apoiados pelas das facilidades de acesso oferecidas pela Internet e pelos avanços das tecnologias de comunicação, têm favorecido a criação de novos ambientes educacionais.

A utilização de softwares em ambientes educacionais torna-se ainda mais comum em cursos oferecido na modalidade a distância, ou EAD. Tendo em vista a grande difusão dessa modalidade de ensino no Brasil, o desenvolvimento de pesquisas envolvendo esses cursos tornouse importante no contexto do sistema de educação no país. Atualmente, o Brasil é um dos países que mais cresce no número de cursos oferecidos na modalidade EAD [1]. Várias iniciativas públicas formam desenvolvidas com o objetivo de incentivar a difusão desta modalidade de ensino. Dentre estas iniciativas pode-se destacar a criação do Programa Universidade Aberta do Brasil (UAB), E-tec Brasil, TV Escola, entre outros

Uma característica comum em ambientes computacionais utilizados no contexto educacional refere-se a sua capacidade de coletar e armazenar uma grande quantidade de dados sobre os estudantes. Esses dados são bastante amplos, variando desde registros de acesso, interações diversas com o sistema até dados com ricos significados semânticos tais como as mensagens em fóruns e chats) [2].

É importante ressaltar que a simples criação de vastas bases de dados torna-se pouco útil sem a disponibilização de ferramentas adequadas para sua análise e interpretação de forma otimizada. Algumas plataformas de apoio ao ensino disponibilizam ferramentas simples de relatório que permitem a extração de algumas informações sobre atividades desenvolvidas por estudantes. Entretanto, é difícil para professores extrair delas informações úteis que apóiem um acompanhamento efetivo dos estudantes [3][4][5].

As limitações dos AVA na disponibilização de informações podem levar a dificuldades no desenvolvimento de atividades de acompanhamento e monitoramento do processo de ensino-aprendizagem dos estudantes nesses ambientes [3][5].

Esse fato é relevante tendo em vista que um aspecto importante para a efetividade de experiências de ensino é a capacidade de professores em monitorar o processo de aprendizagem e tomar decisões com base em eventos observados [5][6]. Desta maneira, o processo de monitoramento e avaliação constitui um diferencial qualitativo entre cursos em um ambiente EAD [6]. Além disso, um professor que monitora os eventos que ocorrem em um AVA teria uma condição privilegiada para tomar decisões.

Entretanto, atualmente este cenário hipotético ainda está muito longe da realidade nas instituições de ensino [5]. Eleutério e Bortolozzi [7] lembram que em cursos que possuem turmas com grande quantidade de estudantes, comuns na modalidade EAD, essa tarefa é ainda mais desafiadora aos professores.

Logo, professores interessados em informações relevantes sobre $\mathrm{o}$ andamento do curso geralmente podem ter apenas uma visão geral dos conteúdos que os estudantes acessaram ou das discussões em que esses se envolveram. Diante disso, o desenvolvimento de ferramentas que possam manipular de maneira significativa grandes volumes de informações sobre os estudantes apresenta-se como um desafio a comunidade de pesquisa de Informática em Educação.

Identificar as informações relevantes em uma base de dados dessa natureza não é uma tarefa simples e não há relatos de modelos que definem claramente esse processo. $\mathrm{O}$ professor que deseja obter informações a respeito do desempenho dos estudantes enfrentará um conjunto de tabelas e dados que, a priori, são de difícil interpretação. Esse professor poderia ser motivado pelas seguintes perguntas:

- Como acompanhar efetivamente um estudante?

- Como verificar se os estudantes estão interagindo entre si?

- Como identificar estudantes com problemas de desempenho?

- Como identificar estudantes desmotivados ou prestes a abandonar o curso?

Haveria uma forma de auxiliar um professor interessado em possíveis respostas para as questões acima? Este trabalho apresenta um estudo preliminar do uso de algumas técnicas de Mineração de Dados em uma base de dados do AVA Moodle [8]. Busca-se, a longo prazo, definir um modelo de análise, inferência ou previsão do comportamento dos aprendizes. Busca-se, a curto prazo, identificar quais parâmetros dessa base dados possibilitariam uma inferência sobre desempenho futuro dos estudantes. Cabe ressaltar que, no contexto deste trabalho, o termo "desempenho" ou "desempenho acadêmico" referese ao resultado final em termos de nota ou conceito obti- 
do pelos estudantes em uma disciplina ou curso

A sequência deste documento está estruturado em 6 seções, numeradas de 2 a 7 . Na Seção 2, analisam-se os trabalhos correlatos. Na seção 3, apresentam-se os critérios utilizados para definição do conjunto de atributos para representação de estudantes. Na seção 4 e 5, são apresentadas, respectivamente, a contextualização e descrição dos experimentos realizados. Na Seção 6, os dados coletados são apresentados em tabelas e analisados. Finalmente, na Seção 7, apresentam-se as conclusões e perspectivas de continuidade deste trabalho

\section{Trabalhos Relacionados}

Mineração de Dados é definida como o processo de descoberta de padrões a partir de um conjunto de dados [9]. Além disso, os padrões descobertos precisam revelar alguma novidade e serem potencialmente úteis de forma a trazer algum benefício para o usuário ou tarefa a ser desenvolvida [10].

Nos últimos anos, diversas técnicas de mineração de dados têm sido utilizadas no contexto educacional. Dentre as técnicas mais utilizadas, destacam-se as que se enquadram no grupo "Previsão"[11].

Em linhas gerais, existem três tipos de técnicas de previsão: classificação, regressão e estimativa de densidade. Para cada técnica de previsão, as variáveis de entrada podem representar categorias ou valores contínuos, sendo que o grau de efetividade dos métodos é fortemente dependente do tipo de variáveis de entrada utilizadas.

A técnica de previsão utilizada neste trabalho é conhecida como classificação. Técnicas de classificação têm por objetivo desenvolver modelos que possam inferir um aspecto particular dos dados (variável a ser prevista) através de alguma combinação de outros aspectos destes dados (variáveis preditoras) [9]. No contexto deste trabalho, a variável a ser prevista é o resultado ou nota final dos estudantes e as variáveis preditoras são os atributos propostos no Quadro 1, apresentado a seguir, na Seção 3 .

O procedimento utilizado para aplicação de técnicas de Mineração de Dados em ambientes educacionais não difere muito da aplicação dessas técnicas em áreas tradicionais como, por exemplo, comércio eletrônico, genética e medicina. Entretanto, algumas características singulares relativas à área educacional requerem a adaptação de algumas técnicas para o problema específico a ser tratado [12].

Em muitas situações de utilização de técnicas de mineração de dados em contextos educacionais, o volume de dados disponíveis pode ser considerado pequeno e o número de atributos grandes, quando comparados com outras áreas que fazem uso de Mineração de Dados [13].
Esse é o caso deste trabalho em que, conforme descrito abaixo na contextualização do estudo, para a realização de experimentos foi utilizada uma base de dados contendo 140 estudantes.

Técnicas de Mineração de Dados educacionais têm sido frequentemente utilizadas para: i) fornecer suporte e mensagens de feedback a professores; ii) recomendações a estudantes; iii) identificação de grupos de estudantes com características comuns; iv) previsão de desempenho ou risco de evasão [1].

Romero et al. [3] apresentaram um estudo evidenciando as possibilidades de utilização de diversas técnicas de Mineração de Dados tais como, classificação, agrupamento, regras de associação para a extração de informações relevantes sobre estudantes no contexto educacional.

A aplicação da CMC (Combination of Multiple Classifiers) para classificar estudantes de acordo com a previsão de nota final e demonstrada em [14]. Segundo esses autores, essa técnica apresentou melhores resultados quando comparada a aplicação de classificadores individualmente.

Nesta linha, outros trabalhos foram desenvolvidos com o objetivo de comparar o desempenho de diversas técnicas de Mineração de Dados aplicadas a problemas de previsão de desempenho [15][16][5] e risco de evasão [17]. Os resultados relatados nessas pesquisas demonstram que diversas técnicas de mineração podem ser utilizadas com sucesso no contexto educacional para previsão de desempenho ou risco de evasão.

O trabalho de Maia et al. [18] destaca-se dentre os trabalhos que tratam a previsão de desempenho pela abordagem utilizada nesta pesquisa. Nesse trabalho, as inferências sobre desempenho futuro de estudantes em disciplinas de um curso de graduação são realizadas a partir das notas obtidas em disciplinas já cursadas. Os autores apresentaram um modelo de representação de aprendizes baseado na teoria dos grafos. Nesse modelo, os alunos e as disciplinas foram modelados como nós e a relação entre eles como arestas de um grafo. A partir de relações de similaridade obtidas por meio do grafo, modelos de crescimento baseados em redes complexas foram capazes de prever a evolução do grafo e, consequentemente, as notas obtidas pelos alunos.

Utilizando pesquisas recentes envolvendo previsão de desempenho, Hämäläinen e Vinni [13] realizaram um estudo para verificar as taxas de acurácia obtidas nestas pesquisas. Os referidos autores destacam que devido às características particulares dos estudos existem variações entre os resultados observados. Porém, destacam que a média de acurácia foi de $72 \%$ entre as pesquisas consideradas. 
Como o foco dos trabalhos destacados acima é a comparação de diferentes algoritmos ou técnicas de aprendizagem de máquina, os autores utilizaram um conjunto restrito de atributos. Este conjunto de atributos não reflete todos os aspectos de interação, atividades e tarefas normalmente desenvolvidas por um estudante em um AVA.

Algumas pesquisas têm relatado o uso, com relativo sucesso, a utilização de técnicas de mineração de dados para inferir a relação existente entre o estado emocional de estudantes e o desempenho apresentado por esses estudantes [19][20].

Utilizar técnicas de Mineração de Dados para identificar quando um estudante está tentando manipular o sistema foi o objetivo do trabalho de Baker et al. [21]. Um exemplo de tentativa de manipular é o fato de um estudante solicitar repetidas vezes ajuda do ambiente computacional antes mesmo de tentar resolver a questão.

Levando-se em consideração a natureza social do aprendizado, algumas pesquisas [4][22][23] têm focado no estudo de como estudantes participam e interagem em um ambiente de aprendizagem virtual.

Destaca-se nesta abordagem de pesquisa o trabalho de Rabbany et al. [23] que investigaram a importância da análise de redes sociais, enfocando a utilização da técnica chamada de "mineração de comunidades". Essa técnica foi utilizada para o estudo e descoberta de estruturas relevantes em fóruns de discussão. O referido trabalho envolveu o desenvolvimento de uma aplicação que permite a visualização dos participantes em um fórum de discussão, suas interações, destacando diferentes papéis assumidos pelos estudantes.

\section{Representação de Estudantes em um AVA}

Para a obtenção de inferências relativas ao desempenho acadêmico futuro de estudantes em um curso EAD utilizando em AVA é necessário, primeiramente, escolher um conjunto de atributos que representem adequadamente esses estudantes.

Neste trabalho, uma referência fundamental utilizada para definir que atributos selecionar para representar estudantes em um curso EAD foi a "Teoria de Interação em Educação a Distância" [24]. Essa teoria destaca três tipos de interação relevantes:

- Entre o estudante e o conteúdo ou objeto de estudo

- Entre o estudante e o especialista que elaborou o material em questão ou algum outro especialista atuando como instrutor
- $\quad$ Entre o estudante e outros estudantes, sozinho ou em grupo, com ou sem a presença em tempo real de um instrutor.

A partir desses tipos de interação relevantes, escolheram-se três dimensões para representar os estudantes em um AVA: perfil de uso do AVA, interação estudanteestudante e interação bidirecional estudante-professor. Essas três dimensões são detalhadas a seguir:

- Primeira Dimensão: Perfil de uso do AVA: nesta dimensão o objetivo é identificar dados que representem aspectos de planejamento, organização e gestão do tempo do estudante para a realização do curso. Para isso definiu-se indicadores gerais de quantidade e tempo médio de acessos aos recursos do AVA. Foram incluídos também atributos que representem atividades rotineiras e regulares dos acessos dos aprendizes.

- Segunda Dimensão: Interação EstudanteEstudante: com esta dimensão pretende-se verificar se os estudantes interagem entre si usando as ferramentas disponíveis, como fóruns, chats, envio ou recebimento de mensagens. Espera-se, com estes atributos, identificar a existência de colaboração e cooperação entre estudantes. Fato esse denominado por Schrire [25] como "aprendendo com os outros" (em inglês learning with others).

- Terceira Dimensão: Interação bidirecional Estudante-Professor: nesta dimensão o objetivo é averiguar como professores ou tutores interagem com estudantes no contexto do AVA. Este tipo de interação tem sua importância destacada por Holliman e Scanlon [26]. Esses autores ressaltam que professores ou tutores têm um papel fundamental no sentido de facilitar e incentivar a colaboração entre estudantes.

Considerando-se as três dimensões apresentadas acima, selecionou-se um conjunto de atributos associados a cada dimensão e que poderiam ser extraídos da base de dados de um AVA Moodle [8]. O Quadro 1 apresenta esses atributos e sua relação com as três dimensões apresentadas. O primeiro atributo "id_estudante" representa o código único de identificação do estudante no AVA. Esse atributo não está relacionado com nenhuma das três dimensões e foi incluído para possibilitar a identificação e busca de informações adicionais sobre o estudante, caso necessário. $\mathrm{O}$ atributo "resultado_final" representa a nota ou conceito final obtido pelo estudante no curso ou disciplina e também não está associado a nenhuma das três dimensões. 
Gottardo, E. at al. Estimativa de Desempenho Acadêmico de Estudantes: Análise da Aplicação de Técnicas de Mineração de Dados em Cursos a Distância

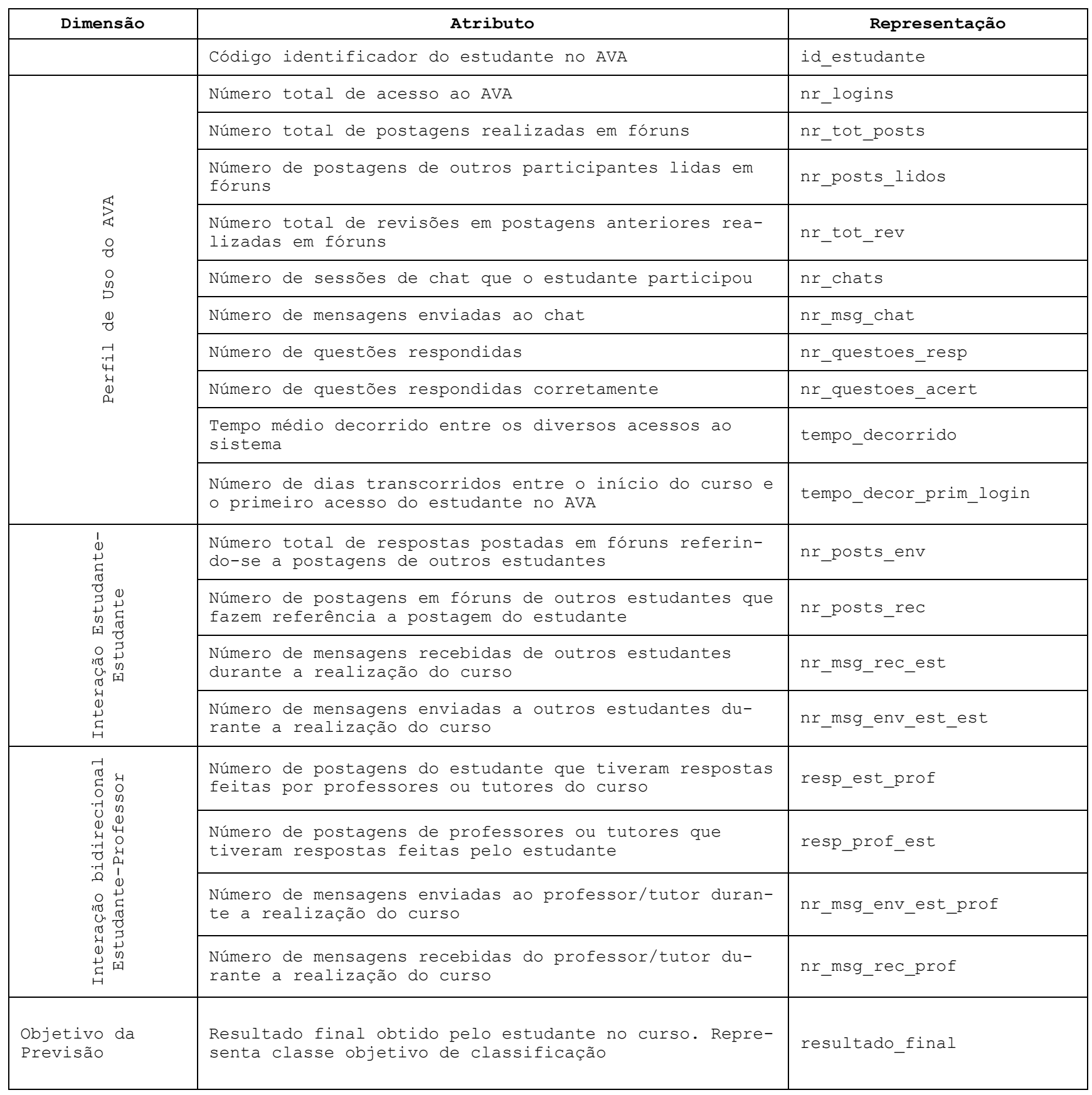

Quadro 1: Atributos selecionados para representação de estudantes em um AVA

\section{Contextualização dos Experimentos}

Com o objetivo de verificar a adequação do conjunto de atributos propostos, realizaram-se experimentos com uma base de dados do AVA Moodle [8], contendo informações de estudantes em um curso realizado a distância.

Considerando-se que o foco deste trabalho refere-se à estimativa do resultado ou nota final, não foram conside- rados estudantes desistentes, tendo em vista a indisponibilidade do resultado final nesses casos. Além disso, a evasão tem relação com fatores específicos como, por exemplo, ambiente sócio-econômico e público-alvo de cursos, tendo sido foco exclusivo de alguns trabalhos [17][22].

Selecionou-se a partir da base de dados Moodle [8] uma disciplina, considerando-se os seguintes requisitos: 
- Maior quantidade de estudantes que concluíram a disciplina

- Maior quantidade de oferta da disciplina para turmas diferentes

- Disponibilidade do resultado de avaliações do conhecimento dos estudantes e

- Maior número de recursos do AVA utilizados.

Baseando-se nos critérios elencados acima, selecionou-se uma disciplina com duas turmas já encerradas, totalizando uma população de 140 estudantes concluintes. Foram então desenvolvidos procedimentos para extração do conjunto de atributos selecionados para representar os estudantes, ilustrados no Quadro 1, a partir do banco de dados do Moodle [8].

É importante observar que o último atributo do Quadro 1, "Resultado_Final", representa a classe objetivo do processo de classificação. Originalmente, este atributo é representado por valores contínuos. Entretanto, algumas técnicas de classificação requerem que o atributo que representa a classe seja um valor discreto.

Para viabilizar a utilização de alguns tipos de métodos e também para facilitar a interpretação dos resultados, foi realizado procedimento de transformação deste atributo, descrito a seguir.

Optou-se por dividir os estudantes em três classes chamadas de "A", "B" e "C" de acordo com a nota obtida no curso. Na classe "A" e "C" serão alocados os estudantes com as notas mais altas e mais baixas, respectivamente. Os demais estudantes farão parte da classe "B".

Analisando-se as notas dos estudantes das turmas selecionadas para o presente trabalho, verificou-se que a distribuição dessas notas aproximam-se de uma curva normal. Esse fato pode ser comprovado observando-se a Figura 1 que apresenta um histograma da distribuição das notas dos estudantes selecionados, gerado pela ferramenta Weka [9]. No eixo horizontal desse gráfico são apresentadas as notas dos estudantes, divididas em nove intervalos iguais, com a média geral 82 ao centro. $\mathrm{O}$ eixo vertical desse gráfico representa o número de estudantes em cada um dos intervalos de distribuição das notas.

Tomando-se como base uma distribuição normal, inicialmente, na classe "B" foram alocados os estudantes cujas notas situem-se em aproximadamente $80 \%$ da área central da curva normal. Os demais estudantes foram distribuídos nas classes "C" e "A", representando, respectivamente, aproximadamente $10 \%$ da área inferior e superior da curva normal.

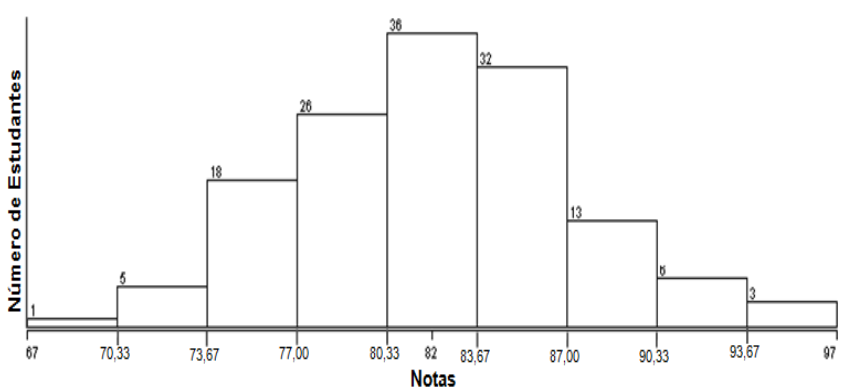

Figura 1: Histograma de distribuição das notas dos estudantes

A Figura 2 apresenta graficamente uma curva normal com a distribuição das classes levando-se em consideração o método descrito acima. A região central dessa figura (destacada em cinza) compreende aproximadamente $80 \%$ da área total e representa a classe "B". Nas extremidades da curva (cor branca) localizam-se os estudantes das classes " $\mathrm{C}$ " e "A". Os números apresentados no eixo horizontal representam as notas que delimitam as classes, com o valor médio global das notas (82) ao centro. O eixo vertical representa o número de estudantes.

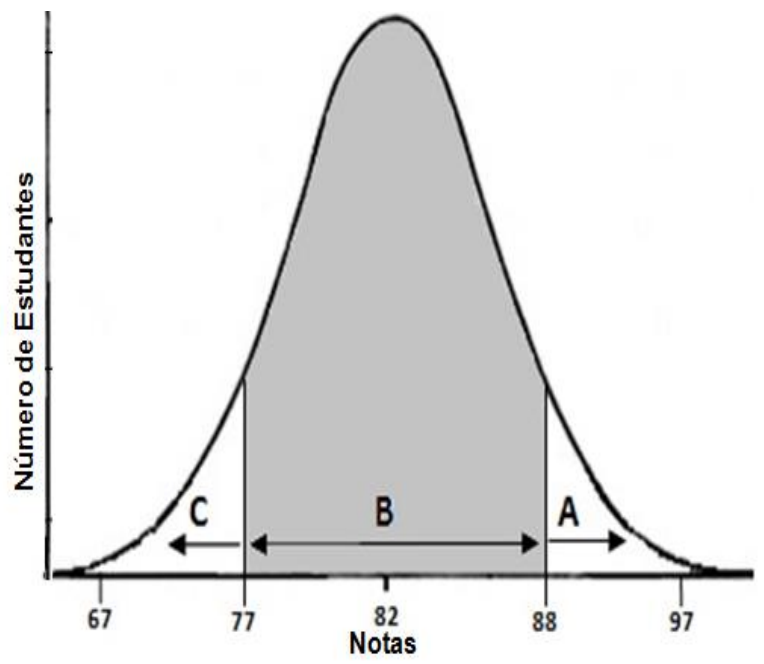

Figura 2: Visualização da distribuição das classes

$\mathrm{Na}$ Tabela 1, as características individuais das três classes são destacadas. Nessa tabela são apresentados o título e a descrição das classes, bem como o número de estudantes e o intervalo de notas em cada uma das três classes criadas.

Além do número de classes, o número de estudantes em cada classe é outro parâmetro que pode influenciar os resultados do processo de classificação. Conforme descrito anteriormente, optou-se por agrupar em cada uma das classes " $\mathrm{A}$ " e "C" aproximadamente $10 \%$ dos estudantes com as maiores e menores notas, respectivamente. A identificação desses dois grupos específicos de estudantes poderia ser considerada importante para um pro- 
fessor interessado em investigar possíveis fatores que contribuem para a um bom ou mau desempenho. É importante observar que o número de classes e o percentual de estudantes em cada classe poderia ser alterado buscandose atingir objetivos específicos da investigação conduzida.

\begin{tabular}{llll}
\hline $\begin{array}{c}\text { Título } \\
\text { da } \\
\text { Classe }\end{array}$ & \multicolumn{1}{c}{ Descrição } & $\begin{array}{c}\text { Número de } \\
\text { Estudantes }\end{array}$ & $\begin{array}{l}\text { Intervalo } \\
\text { de Notas }\end{array}$ \\
\hline A & $\begin{array}{l}\text { Alunos com } \\
\text { desempenho } \\
\text { superior }\end{array}$ & 16 & $\begin{array}{l}\text { Maior que } \\
88\end{array}$ \\
B & $\begin{array}{l}\text { Alunos com } \\
\text { desempenho } \\
\text { intermediário }\end{array}$ & 109 & $\begin{array}{l}\text { Entre } 77 \\
\text { e 88, } \\
\text { inclusive }\end{array}$ \\
C & $\begin{array}{l}\text { Alunos com } \\
\text { desempenho } \\
\text { inferior }\end{array}$ & 15 & $\begin{array}{l}\text { Menor que } \\
77\end{array}$ \\
\hline
\end{tabular}

Tabela 1: Distribuição das classes obtidas pelo processo de discretização.

\section{Experimentos Realizados}

A geração de inferências ou estimativas relativas ao desempenho acadêmico de estudantes tem relação direta com os objetivos deste trabalho. Buscando-se atingir esses objetivos, um estudo com três experimentos foram realizados utilizando-se o conjunto de dados descrito na seção anterior. Nesses experimentos, procurou-se identificar diferentes cenários em que o conjunto de atributos propostos para representação de estudantes poderia ser utilizado para a geração de estimativas de desempenho acadêmico.

Para o desenvolvimento dos experimentos foram utilizados os algoritmos de classificação RandomForest e MultilayerPerceptron [9] disponíveis na ferramenta Weka [9]. A escolha desses algoritmos baseia-se no fato de que eles implementam técnicas de classificação que apresentam bons resultados e cujo uso tem sido frequente em aplicações relacionadas com a área de mineração de dados [27][9]. Adicionalmente, esses algoritmos ou técnicas de classificação, apresentaram resultados promissores quando aplicados ao contexto educacional [17][28][15].

O algoritmo RandomForest é uma técnica de classificação baseada em árvore de decisão, enquanto que o algoritmo MultilayerPerceptron implementa a técnica conhecida como Redes Neurais Artificiais Diretas (Feedforward Artificial Neural Network) [9].

Para a avaliação dos resultados dos dois algoritmos de classificação será utilizada neste trabalho a medida conhecida como acurácia. A acurácia mede a taxa de acerto global, ou seja, o número de classificações corretas dividido pelo número total de instâncias dos dados a serem classificados. Para a avaliação dos resultados dos algoritmos será utilizada também a técnica conhecida como "confusion matrix" ou matriz de confusão [9]. Utilizandose essa técnica, os resultados da classificação são apresentados como uma matriz bidimensional, com uma linha e coluna para cada classe. Cada elemento da matriz mostra o número de instâncias correta ou incorretamente classificadas considerando-se o conjunto de testes utilizado.

Para o cálculo da acurácia utilizou-se o método conhecido como " $K$ - fold Cross-Validation" [9] que é uma técnica para a estratificação da base dados em um conjunto de treinamento e outro de teste. Estudos relatados sugere-se a adoção do número 10 como valor padrão para o número de partições dos dados - K [9].

No experimento 1, foi considerado o conjunto completo de atributos proposto para representação de estudantes, sem nenhuma transformação ou préprocessamento. Desta maneira, foram utilizados os atributos apresentados no Quadro 1, apenas considerando a aplicação do processo de discretização do atributo "Resultado_Final”, conforme apresentado na Tabela 1.

Nesse primeiro experimento, o algoritmo RandomForest obteve uma taxa de acurácia de $74,3 \%$, ou seja, 104 dos 140 estudantes foram classificados corretamente. O algoritmo MultilayerPerceptron obteve neste experimento uma taxa de acurácia de $80,7 \%$, classificando corretamente 113 dos 140 estudantes. A Tabela 2 e Tabela 3 demonstram a matriz de confusão que apresentam os resultados do processo de classificação obtidos nesse experimento. Para facilitar a interpretação, a diagonal principal da matriz de confusão, que apresenta as classificações corretas, foi destacada com fundo cinza. Esse destaque foi utilizado na sequência deste trabalho nas demais tabelas que apresentam outras matrizes de confusão.

\begin{tabular}{|c|c|c|c|c|}
\hline & & \multicolumn{2}{|c|}{ Classes } & Previstas \\
\hline & & A & B & $\mathrm{C}$ \\
\hline & A & 2 & 14 & 0 \\
\hline \multirow[t]{2}{*}{$\begin{array}{l}\text { Classes } \\
\text { Corretas }\end{array}$} & B & 2 & 100 & 7 \\
\hline & $\mathrm{C}$ & 0 & 13 & 2 \\
\hline
\end{tabular}

Tabela 2: Matriz de Confusão do algoritmo RandomForest no Experimento 1 .

Para o experimento 2, foram utilizados todos os atributos apresentados na Quadro 1, transformados para valores discretos. Discretização é o processo pelo qual os valores de um atributo numérico (ex. idade) são substituí- 
dos por rótulos representando intervalos (ex. 0-10, 11-20, etc) ou conceitos (ex. jovem, adulto, sênior) [29]. Essa alternativa baseia-se no fato de que alguns algoritmos de Mineração de Dados, mesmo os capazes de trabalhar com valores contínuos, podem apresentar melhores resultados quando se utiliza valores discretos [9]. Desta forma, foi executado processo de discretização supervisionado dos atributos da base de dados original, utilizando-se o algoritmo padrão da ferramenta Weka [9]. Este algoritmo foi proposto por Fayyad e Irani [30].

\begin{tabular}{lllll}
\hline & & \multicolumn{3}{c}{ Classes Previstas } \\
\hline & A & B & C \\
& A & 11 & 5 & 0 \\
$\begin{array}{l}\text { Classes } \\
\text { Corretas }\end{array}$ & B & 7 & 95 & 7 \\
& C & 0 & 8 & 7 \\
\hline
\end{tabular}

Tabela 3: Matriz de Confusão do algoritmo MultilayerPerceptron no Experimento 1.

Utilizando os dados discretizados, 108 dos 140 estudantes $(77,1 \%)$ foram classificados corretamente pelo o algoritmo RandomForest. A Tabela 4 apresenta a matriz de confusão que destaca os resultados obtidos pelo algoritmo RandomForest nesse experimento. O algoritmo MultilayerPercepetron neste experimento obteve os mesmos valores na matriz de confusão que algoritmo RandomForest.

\begin{tabular}{|c|c|c|c|c|}
\hline & & \multicolumn{2}{|r|}{ Classes } & Previstas \\
\hline & & A & B & $\mathrm{C}$ \\
\hline & A & 0 & 15 & 0 \\
\hline \multirow[t]{2}{*}{$\begin{array}{l}\text { Classes } \\
\text { Corretas }\end{array}$} & B & 0 & 108 & 1 \\
\hline & $\mathrm{C}$ & 0 & 16 & 0 \\
\hline
\end{tabular}

Tabela 4: Matriz de Confusão do algoritmo RandomForest e MultilayerPercepetron no Experimento 2.

Muitos algoritmos são projetados para identificar os atributos mais apropriados a serem utilizados em suas decisões. Na prática, entretanto, a inclusão de atributos irrelevantes em um conjunto de dados frequentemente tem um impacto negativo [9]. Desta maneira, o experimento 3 foi realizado utilizando-se apenas um subconjunto dos atributos propostos no Quadro 1. Nesse processo, utilizou-se o algoritmo de seleção de atributos supervisionado padrão da ferramenta Weka [9], baseado em Hall [31]. Este algoritmo utiliza abordagem baseada em corre- lação, considerando que um bom conjunto de características contém atributos que apresentam alta correlação com a classe e baixa correlação entre si. A execução desse algoritmo resultou na identificação de um subconjunto de quatro atributos dentre a lista completa apresentada no Quadro1. Esses atributos são:

- NR_MSG_REC_PROF - número de mensagens recebidas do professor/tutor durante a realização do curso.

- NR_QUEST_ACERT - número de questões respondidas corretamente.

- NR_CHATS - número de sessões de chat que o estudante participou.

NR_MSG_CHAT - número de mensagens enviadas ao chat.

Considerando-se apenas a lista dos quatro atributos apresentada acima, foram executados os algoritmos de classificação utilizados neste trabalho. O algoritmo RandomForest obteve acurácia de 73,6\%, classificando corretamente 103 dos 140 estudantes. A matriz de confusão destacada na Tabela 5 fornece informações adicionais sobre os resultados desse experimento.

\begin{tabular}{|c|c|c|c|c|}
\hline & & & Classes & Previstas \\
\hline \multirow{4}{*}{$\begin{array}{l}\text { Classes } \\
\text { Corretas }\end{array}$} & & A & B & $\mathrm{C}$ \\
\hline & A & 7 & 9 & 0 \\
\hline & B & 8 & 94 & 7 \\
\hline & $\mathrm{C}$ & 0 & 13 & 2 \\
\hline
\end{tabular}

Tabela 5: Matriz de Confusão do algoritmo RandomForest no Experimento 3 .

O algoritmo MultilayerPerceptron apresentou taxa de acurácia de $75 \%$, ou seja, 105 dos 140 estudantes foram corretamente classificados. Para visualizar mais detalhes dessa classificação a Tabela 6 demonstra a matriz de confusão que detalha os resultados do algoritmo MultilayerPerceptron neste experimento.

A Tabela 7 apresenta um resumo com os resultados dos três experimentos destacados acima. Nela, o percentual de acurácia global médio e o desvio padrão de 100 execuções são apresentados para cada algoritmo e experimento. Para a estratificação da base de dados em conjuntos de teste e treinamento foi utilizado novamente o método "K-fold Cross-Validation". Nessa tabela foi destacado o melhor resultado, que foi obtido pelo algoritmo MultilayerPerceptron no Experimento 1. Adicionalmente, 
destacou-se a menor taxa de acurácia média obtida pelo algoritmo RandomForest no Experimento 3.

\begin{tabular}{llccc}
\hline & & Classes & Previstas \\
\hline & & A & B & C \\
& A & 2 & 14 & 0 \\
$\begin{array}{l}\text { Classes } \\
\text { Corretas }\end{array}$ & B & 5 & 100 & 4 \\
& & & & \\
& C & 0 & 12 & 3 \\
\hline
\end{tabular}

Tabela 6: Matriz de Confusão do algoritmo MultlayerPerceptron no Experimento 3.

\begin{tabular}{|c|c|c|c|}
\hline Classificador & $\begin{array}{l}\text { Experi- } \\
\text { mento } 1\end{array}$ & $\begin{array}{l}\text { Experi- } \\
\text { mento } 2\end{array}$ & $\begin{array}{l}\text { Experi- } \\
\text { mento } 3\end{array}$ \\
\hline RandomForest & $\begin{array}{l}77,4 \pm \\
7,78\end{array}$ & $\begin{array}{l}77,2 \pm \\
2,99\end{array}$ & $\begin{array}{l}72,7 \pm \\
9,92\end{array}$ \\
\hline $\begin{array}{l}\text { Multilayer- } \\
\text { Perceptron }\end{array}$ & $\begin{array}{l}80,1 \pm \\
8,88\end{array}$ & $\begin{array}{l}77,2 \pm \\
2,99\end{array}$ & $\begin{array}{l}76,9 \pm \\
8,07\end{array}$ \\
\hline
\end{tabular}

Tabela 7: Acurácia média e desvio padrão em 100 execuções dos classificadores utilizados nos três experimentos.

\section{Análise dos Resultados}

Analisando-se o resumo dos resultados dos três experimentos destacados na Tabela 7, percebe-se que as taxas médias de acurácia global variaram entre $80,1 \%$ e $72,7 \%$, podendo ser consideradas expressivas no contexto educacional. Essa afirmação baseia-se no resultado do trabalho de Hämäläinen e Vinni [13]. Conforme destacado na Seção 2, esses autores realizaram um estudo para verificar as taxas de acurácia obtidas em pesquisas que envolvem estimativas de desempenho. Nesse estudo, os referido autores relatam que a média da acurácia foi de $72 \%$ entre as pesquisas consideradas.

Pode-se observar ainda na Tabela 7 , que, em números absolutos, no experimento 1 obtiveram-se os melhores resultados em termos de acurácia. Nesse experimento utilizou-se o conjunto completo de atributos propostos no Quadro 1, sem a aplicação de nenhuma técnica específica. Destaca-se que nos dois classificadores utilizados nesse experimento esta tendência pode ser observada. Isso demonstra que os dois algoritmos utilizados foram eficientes na identificação de possíveis atributos irrelevantes, evitando que isso tenha impacto negativo no resultado final.

Comparando-se os resultados do experimento $1 \mathrm{com}$ os resultados do experimento 2 apresentados na Tabela 7 , pode-se notar que a técnica de discretização utilizada no experimento 2 não resultou em melhorias na taxa de acurácia média.

Com o objetivo de testar a significância estatística dos resultados obtidos, utilizou-se a técnica de teste estatístico T pareado - "pair-wise T-Test" [9] com nível significância de 5\%. Para a realização deste teste foi usado o ambiente Weka Experiment Environment - WEE, disponível na ferramenta Weka.

A partir do resultado do teste "T-pareado", observouse que não existe diferença, considerando o nível de significância de $5 \%$, entre os resultados dos três experimentos apresentados na Tabela 7. Essa constatação aponta para a flexibilidade da solução proposta. Em ambientes que não dispõem de todo o conjunto de atributos apresentados no Quadro 1, poder-se-ia utilizar um conjunto reduzido de atributos. Além disso, em casos aonde o número de estudantes é grande, utilizar um conjunto reduzido de atributos pode ser uma alternativa que poderia ser útil para diminuir o tempo de processamento.

É importante destacar também que entre os dois algoritmos utilizados não houve diferença estatisticamente relevante, considerando o teste "T Pareado" com nível de significância de 5\%. Diante disso, o algoritmo RandomForest poderia ser considerado mais adequado para o contexto educacional. Isso porque, diferentemente do algoritmo MultilayerPerceptron, que é do tipo black-box, a estrutura do padrão da solução encontrada pelo algoritmo RandomForest pode ser facilmente entendida por pessoas através da geração de uma árvore de decisão. Além disso, o algoritmo RandomForest apresenta um tempo de processamento menor.

\section{Conclusões e Trabalhos Futuros}

Acompanhar de maneira detalhada e individual estudantes em cursos EAD tem se mostrado um desafio para profissionais e instituições que atuam nesta modalidade de ensino. É consenso entre os autores consultados durante a realização deste trabalho que a implementação de processos efetivos de acompanhamento dos estudantes tem relação direta com a qualidade dos cursos. Diante disso, esforços têm sido feitos pela comunidade científica no desenvolvimento de soluções tecnológicas que forneçam informações relevantes para auxiliar a gestão do processo de ensino desses cursos.

Neste trabalho investigou-se como os dados armazenados por um AVA poderiam ser transformados em informações potencialmente úteis para apoiar o acompanhamento de estudantes em cursos EAD.

Os resultados obtidos com os experimentos realizados apontam para uma possível viabilidade de se realizar inferências relativas ao desempenho de estudantes. Essas 
inferências poderiam ser úteis para professores: i) acompanhar de maneira individual os estudantes que utilizam um AVA; ii) para definir estratégias pedagógicas específicas que busquem minimizar reprovações.

Mesmo considerando-se um conjunto grande de atributos, obtiveram-se resultados expressivos em termos de acurácia das inferências com os dois algoritmos utilizados. Este resultado torna-se relevante, pois aponta para a possibilidade de utilizar um conjunto amplo de atributos que representem de forma abrangente e generalizável um estudante, considerando a diversidade de cursos EAD existentes.

Os resultados iniciais obtidos nesta pesquisa apontam para a viabilidade de investigações futuras considerandose a aplicação de procedimentos semelhantes em diferentes cursos, buscando verificar ou ampliar a generalização do conjunto de atributos propostos.

Adicionalmente, considera-se relevante o desenvolvimento de estudos que utilizem a possibilidade de definição de custos diferentes para erros de classificação para cada classe oferecidas por algumas técnicas de classificação. Além disso, poderiam ser analisados em futuros trabalhos os resultados dos experimentos apresentados nesta pesquisa em cenários com diferentes números de classes.

Levando-se em conta os resultados obtidos neste trabalho, espera-se que novos esforços sejam feitos em futuras pesquisas na tentativa de dotar os AVAs de recursos inteligentes. Esses recursos poderiam ser de monitoramento e adaptação de conteúdo ou monitoramento dos estudantes na expectativa de identificar casos com risco de desempenho insatisfatório.

Futuros trabalhos poderiam ainda ser desenvolvidos no sentido de avaliar a ampliação do conjunto de atributos propostos, considerando a inclusão de informações relativas às atividades desenvolvidas pelos estudantes fora do AVA. Estas atividades poderiam incluir a utilização de ferramentas específicas de um determinado curso ou mesmo utilizações de Redes Sociais, email, entre outros.

A investigação de detalhes sobre os casos de estudantes em que o processo de classificação falhou completamente, ou seja, um estudante da classe " $A$ " que foi classificado erroneamente como " $\mathrm{C}$ " e vice-versa poderia ser tema de trabalhos futuros. Essa investigação poderá apontar a necessidade de inclusão de novos atributos à lista apresentada neste trabalho para aprimorar a representação de estudantes em um AVA e melhorar o processo de classificação.

\section{Referências}

[1] R.S.J.D. Baker, S. Isotani, A.M.J.B.D. Carvalho. Mineração de Dados Educacionais: Oportunidades para o Brasil. Revista Brasileira de Informática na Educação, vol. 19, no. 2, p. 2-13, 2011.

[2] A. Merceron, K. Yacef. Educational Data Mining: a Case Study. In Proceedings of the 12th International Conference on Artificial Intelligence, p. 467-474, 2005.

[3] C. Romero, S. Ventura, E. García. Data Mining in Course Management Systems: Moodle Case Study and Tutorial. Computers \& Education, no. 51, p. 368-384, 2008.

[4] L.P. Macfadyen, S. Dawson. Mining LMS Data to Develop an 'Early Warning System' for Educators: A Proof of Concept. Computers \& Education, no. 54, p. 588-599, 2010.

[5] V.A. Romero-Zaldivar, A. Pardo, D. Burgos, C.D. Kloos. Monitoring Student Progress Using Virtual Appliances: A Case Study. Computers \& Education, no. 58, p. 1058-1067, 2012.

[6] M. G. Moore, G. Kearsley. Educação a distância: uma visão integrada. São Paulo: Thomson Learning, 2007.

[7] M.A. Eleutério, F. Bortolozzi. Amanda: An ITS for Mediating Asynchronous Group Discussions. Lecture Notes in Computer Science - Intelligent Tutoring Systems , vol. 3220, p. 102-115, 2004.

[8] Moodle (2012), disponível em $<$ http://moodle.org $>$. Acesso em 24/03/2012.

[9] I.H. Witten, E. Frank, M.A. Hall. Data Mining: Practical Machine Learning Tools and Techniques. San Francisco: Morgan Kaufmann, 3 ed, 2011.

[10] U.M. Fayyad, G. Piatesky-Shapiro, P. Smyth. The KDD Process for Extracting Useful Knowledge from Volumes of Data. Comunications of ACM, vol. 39, no. 11, p. 27-34, 1996.

[11] C. Romero, S. Ventura. Educational Data mining: A Survey from 1995 to 2005. Expert Systems with Applications, 33:125-146, 2007.

[12] C. Romero, S. Ventura. Educational Data Mining: A Review of the State of Art. IEEE Transactions on Systems , Man, and Cybernetics Part C: Application and Reviews. vol. 40, no. 6, p. 601-618, 2010. 
[13] W. Hämäläinen, M. Vinni. Classifiers for Educational Data Mining. In: Romero et al. Handbook of Educational Data Mining. Flórida, CRC Press, p. 57-71, 2011.

[14] B. Minaei-Bidgoli, A.D. Kashy, G. Kortemeyer, F.W. Punch. Predicting Student Performance: An Application of Data Mining Methods with the Educational Web-Based System LON-CAPA. $33^{a}$ ASEE/IEEE Frontiers in Education Conference, p. 1-6, 2003.

[15] Z. Ibrahim, D. Rusli. Predicting students' Academic Performance: Comparing Artificial Neural Network, Decision Tree and Linear Regression, In Proceedings of the $21^{\circ}$ Annual SAS Malaysia Forum, Kuala Lumpur, Malaysia, p. 1-6, 2007.

[16] S.B. Kotsiantis. Use of Machine Learning Techniques for Educational Proposes: A Decision Support System for Forecasting Student's Grades. Artificial Intelligence Review, vol.37, no. 4, p. 331-344, 2012.

[17] L.M.B. Manhães, S.M.S. Cruz, R.J.M. Costa, J. Zavaleta, G. Zimbrão. Previsão de Estudantes com Risco de Evasão Utilizando Técnicas de Mineração de Dados. In Anais do XXII SBIEXVII WIE, p. 150-159, 2011.

[18] R.F. Maia, E.M. Spina, S.S. Shimizu. Sistema de Previsão de Desempenho de Alunos para Auxilio a Aprendizagem e Avaliação de Disciplinas. In Anais do XXI SBIE-XVI WIE, 2010.

[19] S. Mcquiggan, B. Mott, J. Lester. Modeling SelfEfficacy in Intelligent Tutoring Systems: An Inductive Approach. User Modeling and UserAdapted Interaction, vol.18, p. 81-123, 2008.

[20] S.K. D'mello, S.D. Craig, A.W. Witherspoon, B.T. Mcdaniel, A.C. Graesser. Automatic Detection of Learner's Affect from Conversational Cues. User Modeling and User-Adapted Interaction, p. 45-80, 2008.

[21] R.S.J.D. Baker, A.T. Corbett, K.R. Koedinger, S.E. Evenson, I. Roll, A.Z. Wagner, M. Naim, J. Raspat, D.J. Baker, J. Beck. Adapting to When Students Game an Intelligent Tutoring System. In Proceedings of the International Conference on Intelligent Tutoring Systems, p. 392-401, 2006.

[22] A.J.C, Kampff. Mineração de Dados Educacionais para a Geração de Alertas em Ambientes Virtuais de Aprendizagem como Apoio à Prática Docente. Tese de Doutorado. Programa de PósGraduação em Informática na Educação, Univer- sidade Federal do Rio Grande do Sul, Porto Alegre, 2009.

[23] R.K. Rabbany, M. Takaffoli, O.R. Zaïane. Analyzing Participation of Students in Online Courses Using Social Network Analysis Techniques. In Proceedings of the Fourth International Conference on Educational Data Mining, p. 22-30, 2011.

[24] M. G. Moore. Three Types of Interaction. The American Journal of Distance Education, 3(2):1-6, 1989.

[25] S. Schrire. Building in Asynchronous Discussion Groups: Going Beyond Quantitative Analysis. Computers \& Education, vol. 46, no. 1, p. 4970, 2006.

[26] R. Holliman, E. Scanlon. Investigating Cooperation and Collaboration in Near Synchronous Computer Mediated Conferences. Computers \& Education, no. 46, p. 322-335, 2006.

[27] J.K. Anil, J. Mao, K.M. Mohiunddin. Artificial Neural Networks: A Tutorial. IEEE Computer Society, 29 (3):31-44, 1996.

[28] C. Márquez-Vera, C. Romero, S. Ventura. Predicting School Failure Using Data Mining. In Proceedings of $4^{a}$ International Conference on Educational Data Mining, p. 271-275, 2011.

[29] J. Han, M. Kamber. Data Mining: Concepts and Techniques.California, 2.ed. Morgan Kaufmann, 2006.

[30] U. M. Fayyad, K.B. Irani. Multi-interval Discretization of Continuous Valued Attributes for Classification Learning. In Thirteenth International Joint Conference on Artificial Intelligence, p. 1022-1027, 1993.

[31] M. A. Hall. Correlation-based Feature Subset Selection for Machine Learning. The University of Waikato, Hamilton, New Zealand, 1998. 